\title{
Dietary Intake of Advanced Glycation End Products (AGEs) and Mortality among Individuals with Colorectal Cancer
}

\author{
Ziling Mao ${ }^{1,2}{ }^{\oplus}$, Elom K. Aglago ${ }^{3}$, Zhiwei Zhao ${ }^{1}$, Casper Schalkwijk ${ }^{4}$, Li Jiao ${ }^{5}\left({ }^{\oplus}\right.$, Heinz Freisling ${ }^{3}{ }^{(}$, \\ Elisabete Weiderpass ${ }^{3}{ }^{(}$, David J. Hughes ${ }^{6}{ }^{\circ}$, Anne Kirstine Eriksen ${ }^{7}$, Anne Tjønneland ${ }^{7}$, Gianluca Severi ${ }^{8,9}$, \\ Joseph Rothwell ${ }^{8}$, Marie-Christine Boutron-Ruault ${ }^{8}$, Verena Katzke ${ }^{10}{ }^{\text {, Rudolf Kaaks }}{ }^{10}$, \\ Matthias B. Schulze ${ }^{11,12}{ }^{\circledR}$, Anna Birukov ${ }^{11}{ }^{\mathbb{C}}$, Vittorio Krogh ${ }^{13}{ }^{\mathbb{D}}$, Salvatore Panico ${ }^{14}$, Rosario Tumino ${ }^{15,16}$, \\ Fulvio Ricceri ${ }^{17}$, H. Bas Bueno-de-Mesquita ${ }^{18}$, Roel C. H. Vermeulen ${ }^{19}$, Inger T. Gram ${ }^{20}$, Guri Skeie ${ }^{20}{ }^{(D}$, \\ Torkjel M. Sandanger ${ }^{20}$, J. Ramón Quirós ${ }^{21}$, Marta Crous-Bou ${ }^{22,23}$, Maria-Jose Sánchez ${ }^{24,25,26,27}$, \\ Pilar Amiano 28,29,30, María-Dolores Chirlaque 30,31 , Aurelio Barricarte Gurrea ${ }^{30,32,33}$, Jonas Manjer ${ }^{34}$, \\ Ingegerd Johansson ${ }^{35}\left(\mathbb{D}\right.$, Aurora Perez-Cornago ${ }^{36}$, Mazda Jenab ${ }^{3}(\mathbb{D})$ and Veronika Fedirko $1,37, * \mathbb{C}$
}

\section{check for}

updates

Citation: Mao, Z.; Aglago, E.K.; Zhao, Z.; Schalkwijk, C.; Jiao, L.; Freisling, H.; Weiderpass, E.; Hughes, D.J.; Eriksen, A.K.; Tjønneland, A.; et al. Dietary Intake of Advanced Glycation End Products (AGEs) and Mortality among Individuals with Colorectal Cancer. Nutrients 2021, 13, 4435. https:// doi.org/10.3390/nu13124435

Academic Editor: Leni Rose Rivera

Received: 16 November 2021 Accepted: 6 December 2021 Published: 10 December 2021

Publisher's Note: MDPI stays neutral with regard to jurisdictional claims in published maps and institutional affiliations.

Copyright: (C) 2021 by the authors. Licensee MDPI, Basel, Switzerland. This article is an open access article distributed under the terms and conditions of the Creative Commons Attribution (CC BY) license (https:/ / creativecommons.org/licenses/by/ $4.0 /$ )
1 Department of Epidemiology, Rollins School of Public Health, Emory University, Atlanta, GA 30322, USA zim12@pitt.edu (Z.M.); zhiwei.zhaoxmu@hotmail.com (Z.Z.)

2 Department of Epidemiology, Graduate School of Public Health, University of Pittsburgh, Pittsburgh, PA 15261, USA

3 Section of Nutrition and Metabolism, International Agency for Research on Cancer, World Health Organization (IARC-WHO), 69372 Lyon, France; AglagoE@fellows.iarc.fr (E.K.A.); Freislingh@iarc.fr (H.F.); Director@iarc.fr (E.W.); jenabm@iarc.fr (M.J.)

4 Department of Internal Medicine, School for Cardiovascular Diseases (CARIM), Maastricht University, 6229ER Maastricht, The Netherlands; c.schalkwijk@maastrichtuniversity.nl

5 Baylor College of Medicine, 2002 Holcombe Blvd, Houston, TX 77030, USA; jiao@bcm.edu

6 Cancer Biology and Therapeutics Group, School of Biomolecular and Biomedical Science, UCD Conway Institute, University College Dublin, Belfield, D04 V1W8 Dublin, Ireland; david.hughes@ucd.ie

7 Danish Cancer Society Research Center, Diet, Genes and Environment Nutrition and Biomarkers (NAB), Strandboulevarden 49, DK-2100 Copenhagen, Denmark; ake@cancer.dk (A.K.E.); annet@cancer.dk (A.T.)

8 CESP (UMR1018), Faculté de Médecine Université Paris-Saclay, Inserm, Gustave Roussy, 94805 Villejuif, France; gianluca.severi@inserm.fr (G.S.); joseph.rothwell@gustaveroussy.fr (J.R.); marie-christine.boutron@gustaveroussy.fr (M.-C.B.-R.)

9 Department of Statistics, Computer Science and Applications (DISIA), University of Florence, 50121 Florence, Italy

10 Division of Cancer Epidemiology, German Cancer Research Center (DKFZ), 69120 Heidelberg, Germany; V.Katzke@Dkfz-Heidelberg.de (V.K.); r.kaaks@Dkfz-Heidelberg.de (R.K.)

11 Department of Molecular Epidemiology, German Institute of Human Nutrition Potsdam-Rehbruecke, 14558 Nuthetal, Germany; mschulze@dife.de (M.B.S.); Anna.Birukov@dife.de (A.B.)

12 Institute of Nutritional Science, University of Potsdam, 14558 Nuthetal, Germany

13 Epidemiology and Prevention Unit, Fondazione IRCCS Istituto Nazionale dei Tumori di Milano, Via Venezian, 20133 Milan, Italy; vittorio.krogh@istitutotumori.mi.it

14 Dipartmento Di Medicina Clinica E Chirurgia, Federico II University, 80131 Naples, Italy; salvatorepanico2@gmail.com

15 Cancer Registry and Histopathology Department, Provincial Health Authority (ASP 7), 97100 Ragusa, Italy; rtuminomail@gmail.com

16 Hyblean Association for Epidemiological Research, AIRE-ONLUS, 97100 Ragusa, Italy

17 Department of Clinical and Biological Sciences, University of Turin, 10043 Turin, Italy; fulvio.ricceri@unito.it

18 Centre for Nutrition, Prevention and Health Services, National Institute for Public Health and the Environment (RIVM), 3720 Bilthoven, The Netherlands; basbuenodemesquita@gmail.com

19 Division of Environmental Epidemiology, Institute for Risk Assessment Sciences, Utrecht University, 80178 Utrecht, The Netherlands; R.C.H.Vermeulen@uu.nl

20 Department of Community Medicine, University of Tromsø, The Arctic University of Norway, 9010 Tromsø, Norway; inger.gram@uit.no (I.T.G.); guri.skeie@uit.no (G.S.); torkjel.sandanger@uit.no (T.M.S.)

21 Public Health Directorate, 33080 Asturias, Spain; joseramon.quirosgarcia@asturias.org

22 Unit of Nutrition and Cancer, Cancer Epidemiology Research Program, Catalan Institute of Oncology (ICO)—Bellvitge Biomedical Research Institute (IDIBELL), L'Hospitalet de Llobregat, 08908 Barcelona, Spain; marta.crous@iconcologia.net

23 Department of Epidemiology, Harvard T.H. Chan School of Public Health, Boston, MA 02115, USA

24 Escuela Andaluza de Salud Pública (EASP), 18011 Granada, Spain; mariajose.sanchez.easp@juntadeandalucia.es 
25 Instituto de Investigación Biosanitaria ibs.GRANADA, 18012 Granada, Spain

26 Centro de Investigación Biomédica en Red de Epidemiología y Salud Pública (CIBERESP), 28029 Madrid, Spain

27 Department of Preventive Medicine and Public Health, University of Granada, 18012 Granada, Spain

28 Ministry of Health of the Basque Government, Sub Directorate for Public Health and Addictions of Gipuzkoa, 20013 San Sebastian, Spain; epicss-san@euskadi.eus

29 Epidemiology of Chronic and Communicable Diseases Group, Biodonostia Health Research Institute, 20013 San Sebastian, Spain

30 Spanish Consortium for Research on Epidemiology and Public Health (CIBERESP), Instituto de Salud Carlos III, 28029 Madrid, Spain; mdolores.chirlaque@carm.es (M.-D.C.);

aurelio.barricarte.gurrea@navarra.es (A.B.G.)

31 Department of Epidemiology, Regional Health Council, IMIB-Arrixaca, Murcia University, 30100 Murcia, Spain

32 Navarra Public Health Institute, 31003 Pamplona, Spain

33 Navarra Institute for Health Research (IdiSNA), 31008 Pamplona, Spain

34 Department of Surgery, Skåne University Hospital Malmö, Lund University, SE-221 00 Malmö, Sweden; jonas.manjer@med.lu.se

35 Department of Epidemiology and Clinical Medicine, Umeå University, SE-901 87 Umeå, Sweden; ingegerd.johansson@umu.se

36 Cancer Epidemiology Unit, Nuffield Department of Population Health, University of Oxford, Oxford OX3 7LF, UK; aurora.perez-cornago@ndph.ox.ac.uk

37 MD Anderson Cancer Center, Department of Epidemiology, 1515 Holcombe Blvd., Unit 1340, Houston, TX 77030, USA

* Correspondence: vfedirko@mdanderson.org; Tel.: +1-832-750-5957

Abstract: Advanced glycation end-products (AGEs) may promote oxidative stress and inflammation and have been linked to multiple chronic diseases, including cancer. However, the association of AGEs with mortality after colorectal cancer (CRC) diagnosis has not been previously investigated. Multivariable Cox proportional hazards models were used to calculate hazard ratios and corresponding 95\% confidence intervals for associations between dietary intake of AGEs with CRC-specific and all-cause mortality among 5801 participant cases diagnosed with CRC in the European Prospective Investigation into Cancer and Nutrition study between 1993 and 2013. Dietary intakes of AGEs were estimated using country-specific dietary questionnaires, linked to an AGE database, that accounted for food preparation and processing. During a median of 58 months of follow-up, 2421 cases died (1841 from CRC). Individually or combined, dietary intakes of AGEs were not associated with all-cause and CRC-specific mortality among cases. However, there was a suggestion for a positive association between AGEs and all-cause or CRC-specific mortality among CRC cases without type II diabetes (all-cause, $P_{\text {interaction }}=0.05$ ) and CRC cases with the longest follow-up between recruitment and cancer diagnosis $\left(C R C\right.$-specific, $P_{\text {interaction }}=0.003$; all-cause, $\left.P_{\text {interaction }}=0.01\right)$. Our study suggests that pre-diagnostic dietary intakes of AGEs were not associated with CRC-specific or all-cause mortality among CRC patients. Further investigations using biomarkers of AGEs and stratifying by sex, diabetes status, and timing of exposure to AGEs are warranted.

Keywords: advanced glycation end-products; dietary advanced glycation end-products; all-cause mortality; colorectal cancer mortality

\section{Introduction}

Colorectal cancer (CRC) is the third leading cause of cancer death worldwide [1]. Large differences in incidence rates across countries and rapid changes in incidence among migrant populations suggest that modifiable environmental factors, including diet and lifestyle, play an important role in CRC etiology [2]. Despite advances in screening and treatment, there is an increasing number of CRC survivors that are at risk for CRC recurrence and death [3]. Multiple lifestyle and dietary factors such as tobacco smoking, obesity, and heavy alcohol intake have been linked to the risk of CRC [4]. However, knowledge of the risk factors affecting survival after CRC diagnosis is limited. It is of interest to 
determine whether modifiable factors may affect cancer survival, particularly those that could be modified by changes in diet or lifestyle.

Advanced glycation end products (AGEs) comprise a large heterogeneous group of compounds derived from a series of irreversible reactions between reducing sugars and free amino groups in amino acids, or oxidation of sugars, lipids, and amino acids [5]. Endogenous AGEs are formed in the human body under physiological conditions, and their formation is enhanced in people with diabetes [6]. A major exogenous source of the pool of AGEs in our body is from the diet $[7,8]$. Foods rich in both fat and protein and cooked at high temperature and with dry heat processing, such as grilling, broiling, frying, or roasting, tend to be the major sources of dietary AGEs [9]. The best characterized dietary AGEs include $\mathrm{N}^{\varepsilon}$-[carboxymethyl]lysine (CML), $\mathrm{N}^{\varepsilon}$-[1-carboxyethyl]lysine (CEL), and $\mathrm{N}^{\delta}$-[5-hydro-5-methyl-4-imidazolon-2-yl]-ornithine (MG-H1) [10].

Experimental studies have suggested that the accumulation of AGEs can increase oxidative stress and inflammation in the tissue through binding to the receptor for advanced glycation end products (RAGEs) [11-16]. Another mechanism through which AGEs induce pathological effects is via the cross-linking of collagen and other proteins, which could contribute to structural and physiologic changes in the cardiovascular system [17,18]. Through the above mechanisms, AGEs may act as a risk factor for aging-related health outcomes [11-13], including reduced survival among cancer patients. In epidemiologic studies, higher AGEs have been associated with various chronic diseases, including some cancers and cardiovascular disease (CVD) [19-24], which are associated with reduced survival. However, the results of previously published observational studies on the association of AGEs with mortality risk were inconsistent [21,25-31], which in part could be due to different populations included in the studies (e.g., patients with diabetes or breast cancer, or older healthy individuals) and different methods for estimating exposure to AGEs. Among these, most studies focused on AGE measurements in plasma [21,26-30], and only two prospective studies $[25,31]$ investigated the associations of dietary AGEs with mortality risk. The first [25] reported a positive association between higher post-diagnosis dietary AGE intake and all-cause, CVD, and breast cancer mortality among breast cancer patients. However, the second study among healthy Japanese adults [31] suggested that dietary AGEs are not associated with higher risk of all-cause mortality. To our knowledge, so far, no prospective studies have investigated the association of dietary AGEs with mortality risk among CRC patients.

Therefore, we examined the associations between pre-diagnostic dietary intakes of the three best characterized AGEs-CML, CEL, and MG-H1-and CRC-specific and all-cause mortality among individuals diagnosed with CRC in the large, multi-center prospective cohort, the European Prospective Investigation into Cancer and Nutrition (EPIC) study. We hypothesized that higher dietary intakes of these AGEs before cancer diagnosis are associated with higher mortality risk.

\section{Methods}

\subsection{Study Population and Data Collection}

The EPIC is a large, multi-center prospective cohort study with more than 520,000 participants. The details and methods of the EPIC study have been reported previously [32,33]. Participating countries include France, Germany, Greece, Italy, The Netherlands, Spain, the United Kingdom, Sweden, Denmark, and Norway. Between 1992 and 1998, standardized lifestyle/personal history questionnaires, anthropometric data, and blood samples were collected from most participants at recruitment, before disease onset or diagnosis.

Individuals who were eligible for the study were recruited from the general population of a specific geographical area, town, or province. Exceptions included the French subcohort and the Utrecht (The Netherlands) sub-cohort: the former is based on members of the health insurance system or state-school employees, while the latter is based on women who underwent screening for breast cancer. In addition, a portion of the Spanish and Italian sub-cohorts included blood donors. In our analysis, we included participants from 
all centers except Greece (excluded due to data restriction issues). Lifestyle questionnaires were used to obtain information on education, physical activity, lifetime alcohol intake, smoking status, and self-reported diabetes mellitus status at baseline. Anthropometric measures were assessed at recruitment, and body mass index (BMI) was computed as weight in kilograms over height in square meters.

The EPIC study was approved by the Ethical Review Board of the International Agency for Research on Cancer (IARC) and the Institutional Review Boards of each participating center. Written consent was obtained from all EPIC participants upon enrollment into the study.

\subsection{Dietary Assessment and Estimation of AGE Intake}

In EPIC, country- or center-specific validated dietary questionnaires (DQs) were completed at baseline, accounting for the usual food intake during the previous 12 months [32]. The Netherlands, Germany, Northern Italy, and France used quantitative DQs. In Spain and Ragusa (Italy), the quantitative DQs were interviewer-administered and structured by meals. Malmö (Sweden) and the UK used semi-quantitative food frequency questionnaires in combination with 7-day and 14-day records, respectively. In Umeå (Sweden), Denmark, Norway, and Naples (Italy), semi-quantitative food frequency questionnaires (FFQs) were used. Harmonization of food groups and portion sizes for quantification was carried out centrally at the IARC [34]. Cooking methods were not included in the DQ and FFQ. We used the most common cooking methods in a given country reported in the 24-hour recalls either in the EPIC calibration study or in national surveys.

Dietary AGEs were estimated using a reference dietary AGE food composition database, which is based on the CML, CEL, and MG-H1 concentrations (in mg/100 g of food) obtained from 190 food items commonly consumed in Europe using ultra-performance liquid chromatography tandem mass-spectrometry analysis [10]. Foods from the reference database were matched to those included in the DQs by name and descriptors, particularly those pertaining to preparation and processing whenever applicable [35]. Generic or multi-ingredient $\mathrm{DQ}$ foods were decomposed into more specific foods or ingredients based on country-specific recipes obtained from previous EPIC projects [34,36]. The EPICspecific AGE composition database was then generated and used to obtain the daily intake (mg/day) of CML, CEL, and MG-H1 per study participant. The validity of these data was further confirmed by assessing the expected associations between higher dietary intakes of any of three AGEs and weight gain after an average of five years of follow-up in the same study population [36].

\subsection{Cancer Ascertainment and Follow-Up}

Cancer data was coded using the International Classification of Disease (ICD)-10 and the second revision of the ICD for Oncology (ICD-O-2). CRC cases included participants who developed colon (C18.0-C18.7), rectal (C19-C20), and overlapping or unspecified origin colorectal tumors (C18.8-C18.9). CRC included colon and rectal cancer cases. Colon cancer included tumors that developed in both the proximal site (C18.0-C18.5: cecum, appendix, ascending colon, hepatic flexure, transverse colon, and splenic flexure) and the distal site (C18.6-C18.7: descending and sigmoid colon).

Incident cancer cases were ascertained through record linkage with regional cancer registries (Denmark, Italy, The Netherlands, Norway, Spain, Sweden, and the United Kingdom; complete up to 2011-2013) or through a combination of methods, including the use of health insurance records, contacts with cancer and pathology registries, and active follow-up through study subjects and their next-of-kin (France and Germany; complete up to 2008 and 2009). Of 6027 identified CRC cases, we excluded individuals who were missing dietary AGE data $(n=99)$, had tumor stage coded as in situ $(n=3)$, had a follow-up time of zero due to cancer diagnosis listed on the death certificate $(n=4)$, or reported extreme total energy intakes (top and bottom $1 \%$ of the total energy intake to estimated energy requirements ratio; $n=120$ ), leaving 5801 CRC cases for the final analytic cohort. 


\subsection{Vital Status Follow-Up}

Vital status follow-up was determined through record linkage with regional and/or national mortality registries (Denmark, Italy, The Netherlands, Spain, and the United Kingdom) or active follow-up (France and Germany). Censoring dates for complete followup were between January 2013 and February 2015. Mortality was coded using ICD-10 (which includes Injuries and Causes of Death), and the outcome was assigned based on the underlying cause of death.

\subsection{Statistical Analyses}

Dietary intakes of CML, CEL, and MG-H1 were natural log (ln)-transformed, and total energy intake was adjusted using the residual method [37]. For energy adjustment, we computed standardized residuals of each of the three AGEs by regressing the lntransformed AGEs on total energy intake, sex, and center and adding back the sex- and center-specific mean to each observation. The combined AGE value was calculated as the sum of the three total energy-adjusted AGEs. The total energy-adjusted AGEs were analyzed separately and combined on a continuous scale per standard deviation (SD) increment and as quintiles of intake across all centers.

Death from CRC was the primary endpoint, and death from any cause was used as a secondary endpoint. Entry time was age at first tumor diagnosis, and exit time was either death or censoring date (lost to or end of follow-up), whichever event occurred first. Cox proportional hazard models were used to calculate hazard ratios (HRs) and $95 \%$ confidence intervals (CIs). The proportional hazard assumptions for all variables in the model were tested with Schoenfeld residuals and included a time-dependent covariate in the Cox model. Two main models were fitted with different sets of adjustments. Model 1 was stratified by center and adjusted for sex, age at diagnosis (continuous, years), tumor stage (I, II, III, IV, missing), and total energy intake (continuous, kcal/day). To determine the final model (Model 2), the following a priori identified covariates were assessed as potential confounders: grade of tumor differentiation (well, moderately, poorly differentiated, unknown), location of primary tumor (colon or rectum), smoking status (never smoker, former smoker, current smoker, unknown), BMI $\left(\mathrm{kg} / \mathrm{m}^{2}\right)$, year of diagnosis, dietary intakes (red and processed meats, fruits and vegetables, dietary calcium, dietary fiber, sugar, dairy, alcohol drinking pattern), physical activity, and type II diabetes based on self-reporting at baseline and ascertainment before cancer diagnosis. These variables were chosen based on previous published evidence showing their associations with CRC incidence or survival and/or AGEs. We evaluated confounding by assessing change $(>10 \%)$ in HRs after including the variables in the model. The final Model 2 was stratified by center and adjusted for year of diagnosis (continuous), location of tumor (colon/rectum), BMI (continuous, $\mathrm{kg} / \mathrm{m}^{2}$ ), smoking status (never, former, current, missing), and type II diabetes (no, yes, missing; defined as being diagnosed with diabetes at baseline or during follow-up). Participants with missing values were included in all analyses, and respective variables were coded with a missing value indicator, unless otherwise specified. $p$-value for trend was calculated, with the median value of each AGE quintile included as a continuous variable in the corresponding models.

To evaluate the linearity of the dose-response associations between continuous intakes of AGEs and risk for CRC-specific and all-cause mortality, non-parametric restricted cubic splines $[38,39]$ were fitted to a Cox proportional hazard model using the SAS macro "lgtphcurv9" [40]. Tests for non-linearity used the likelihood ratio test, comparing the model with only the linear term to the model with both the linear and cubic spline terms [40].

We assessed whether missing tumor stage information influenced the effect estimates using several approaches. The first approach reclassified missing tumor stage values into a separate missing category and adjusted for the stage variable in the final model (included in the primary analysis). Second, a sensitivity analysis was conducted by excluding participants with missing stage information and subsequently by assessing how the results were affected by the missing stage information. Finally, an imputation of missing tumor 
stage values was conducted using the SAS PROC MI procedure as described previously [41]. The multiple imputation method was based on available data for the other covariates in the model and assumed that the stage data was missing at random.

Stratified analyses by categories of potentially biologically relevant effect modifiers (time interval between recruitment and CRC diagnosis, length of follow-up, sex, age at diagnosis, tumor site, grade, and stage, BMI, physical activity, smoking status, alcohol intake, and prevalent and incident diabetes) were conducted. Stratified multivariableadjusted HRs and 95\% CIs were reported per 1 SD increase in CML, CEL, MG-H1, and combined AGEs. A cross-product of AGE as a continuous variable and the covariate of interest as a continuous or categorical variable was included in the model to test for multiplicative statistical interaction; the likelihood ratios based on the models with and without the interaction terms were used to test for statistical significance.

All statistical tests were conducted using SAS version 9.2 (SAS Institute, Cary, NC, USA). $p$-values of $<0.05$ were considered statistically significant.

\section{Results}

\subsection{Patient Characteristics}

Among the 5801 eligible CRC cases, over a mean of 68 (median $=58$ ) months of follow up, 2421 died of any cause (including 1841 from CRC). Selected baseline characteristics of study participants across quintiles of the combined AGEs are shown in Table 1. CRC cases in the highest compared to the lowest quintile of the combined AGEs were less likely to be current smokers and, on average, had lower red meat consumption.

Table 1. Selected baseline characteristics of CRC cases $(N=5801)$ according to quintiles of pre-diagnostic combined ${ }^{a}$ energy-adjusted dietary AGE intake ${ }^{b}$ in the EPIC study.

\begin{tabular}{|c|c|c|c|c|c|c|}
\hline \multirow[b]{2}{*}{ Characteristic } & \multicolumn{6}{|c|}{ Combined ${ }^{\text {a }}$ Dietary AGEs (mg/d) } \\
\hline & $\begin{array}{l}\text { Quintile 1: } \\
\quad<19.79 \\
(N=1160)\end{array}$ & $\begin{array}{c}\text { Quintile 2: } \\
19.79-23.20 \\
(N=1160)\end{array}$ & $\begin{array}{c}\text { Quintile 3: } \\
23.21-26.80 \\
(N=1161)\end{array}$ & $\begin{array}{l}\text { Quintile 4: } \\
26.81-32.26 \\
(N=1160)\end{array}$ & $\begin{array}{c}\text { Quintile 5: } \\
>32.26 \\
(N=1160)\end{array}$ & $p$-Value \\
\hline Age at diagnosis, mean (SD), y & $66.04(7.68)$ & $65.69(8.63)$ & $66.01(8.65)$ & $66.49(8.70)$ & $67.25(8.93)$ & 0.39 \\
\hline Women, $N(\%)$ & $870(75)$ & $709(61)$ & $680(59)$ & $570(49)$ & $491(42)$ & $<0.001$ \\
\hline Year at $\mathrm{dx}$, median (min-max) & $\begin{array}{c}2004 \\
(1993-2013)\end{array}$ & $\begin{array}{c}2005 \\
(1993-2013)\end{array}$ & $\begin{array}{c}2005 \\
(1993-2013)\end{array}$ & $\begin{array}{c}2005 \\
(1993-2013)\end{array}$ & $\begin{array}{c}2005 \\
(1992-2013)\end{array}$ & 0.89 \\
\hline Stage of disease, $N(\%)^{\mathrm{c}}$ & & & & & & $<0.001$ \\
\hline I & $225(19)$ & $217(19)$ & $228(20)$ & $259(22)$ & $226(19)$ & \\
\hline II & $241(21)$ & $224(19)$ & $177(15)$ & $213(18)$ & $172(15)$ & \\
\hline III & $286(25)$ & $304(26)$ & $325(28)$ & $258(22)$ & $250(22)$ & \\
\hline IV & $165(14)$ & $142(12)$ & $128(11)$ & $116(10)$ & $98(8)$ & \\
\hline Location of primary tumor, $N(\%)$ & & & & & & 0.75 \\
\hline Colon & $762(66)$ & $748(65)$ & $766(66)$ & $750(65)$ & $752(65)$ & \\
\hline Rectum & $398(34)$ & $412(35)$ & $395(34)$ & $410(35)$ & $408(35)$ & \\
\hline Smoking status, $N(\%)^{\mathrm{c}}$ & & & & & & $<0.001$ \\
\hline Never & $462(40)$ & $482(42)$ & $462(40)$ & $471(41)$ & $494(43)$ & \\
\hline Former & $364(31)$ & $365(31)$ & $403(35)$ & $410(35)$ & $422(36)$ & \\
\hline Current & $326(28)$ & $301(26)$ & $276(24)$ & $259(22)$ & $224(19)$ & \\
\hline BMI, mean (SD), kg/m² & $26.14(4.33)$ & $26.29(4.21)$ & $26.34(4.16)$ & $26.54(4.16)$ & $26.17(4.26)$ & 0.15 \\
\hline Physical activity ${ }^{\mathrm{d}}, N(\%)^{\mathrm{c}}$ & & & & & & $<0.001$ \\
\hline Inactive & $197(17)$ & $182(16)$ & $187(16)$ & $181(16)$ & $128(11)$ & \\
\hline Moderately inactive & $383(33)$ & $375(32)$ & $351(30)$ & $320(28)$ & $347(30)$ & \\
\hline Moderately active & $446(38)$ & $450(39)$ & $458(40)$ & 458 (39) & $438(38)$ & \\
\hline Active & $85(7)$ & $105(9)$ & $99(9)$ & $103(9)$ & $123(11)$ & \\
\hline Diabetes ${ }^{\mathrm{e}}, N(\%)^{\mathrm{c}}$ & & & & & & $<0.001$ \\
\hline No & $952(82)$ & $943(81)$ & $922(80)$ & $901(78)$ & $881(76)$ & \\
\hline Yes & $105(9)$ & $106(9)$ & $122(11)$ & $111(10)$ & $105(9)$ & \\
\hline Daily dietary intakes & & & & & & \\
\hline Total energy, mean (SD), kcal & $2019.4(584.6)$ & $2098.2(609.6)$ & $2115.0(602.4)$ & $2158.6(628.8)$ & $2155.3(639.2)$ & $<0.001$ \\
\hline
\end{tabular}


Table 1. Cont.

\begin{tabular}{|c|c|c|c|c|c|c|}
\hline \multirow[b]{2}{*}{ Characteristic } & \multicolumn{6}{|c|}{ Combined a Dietary AGEs (mg/d) } \\
\hline & $\begin{array}{c}\text { Quintile 1: } \\
\quad<19.79 \\
(N=1160)\end{array}$ & $\begin{array}{c}\text { Quintile 2: } \\
19.79-23.20 \\
(N=1160)\end{array}$ & $\begin{array}{c}\text { Quintile 3: } \\
23.21-26.80 \\
(N=1161)\end{array}$ & $\begin{array}{c}\text { Quintile 4: } \\
26.81-32.26 \\
(N=1160)\end{array}$ & $\begin{array}{c}\text { Quintile 5: } \\
>32.26 \\
(N=1160)\end{array}$ & $p$-Value \\
\hline Fiber, mean (SD), g & $19.8(6.9)$ & $21.6(6.8)$ & $22.8(7.1)$ & $23.9(7.7)$ & $25.4(8.7)$ & $<0.001$ \\
\hline Dietary calcium, mean (SD), mg & $980.4(440.7)$ & $976.7(408.5)$ & $969.8(378.6)$ & $989.4(375.1)$ & $1003.4(394.1)$ & 0.30 \\
\hline Fruits, mean (SD), g & $220.6(191.4)$ & $223.3(184.7)$ & $225.2(183.0)$ & $222.1(161.4)$ & $202.9(155.0)$ & 0.02 \\
\hline Vegetables, mean (SD), g & $179.9(123.3)$ & $178.7(115.9)$ & $187.0(115.0)$ & $193.7(125.2)$ & $191.7(132.1)$ & 0.007 \\
\hline Red meat, mean (SD), g & $50.9(35.3)$ & $52.7(38.0)$ & $52.7(41.1)$ & $46.5(37.6)$ & $41.9(37.4)$ & $<0.001$ \\
\hline Processed meat, mean (SD), g & $30.9(26.7)$ & $34.5(28.8)$ & $34.6(31.3)$ & $37.8(30.5)$ & $37.2(36.1)$ & $<0.001$ \\
\hline
\end{tabular}

Abbreviations: AGE, advanced glycation end product; BMI, body mass index; CRC, colorectal cancer; CML, Ne-(caroxymethyl)lysine; CEL, Ne-(1-caroxyethyl)lysine; d, day; g, gram; MG-H1, Ne-(5-hydro-5-methyl-4-imidazolon-2-yl)-ornithine; SD, standard deviation; y, years; dx, diagnosis. ${ }^{a}$ Combined AGEs $=\mathrm{CML}+\mathrm{CEL}+\mathrm{MG}-\mathrm{H} 1 .{ }^{\mathrm{b}}$ Total energy-adjusted residuals were computed by fitting a linear regression of the log-transformed intake of AGEs on total energy intake, sex, and center. ${ }^{c}$ The sum of percentages across subgroups did not add up to $100 \%$ due to missing values. ${ }^{\mathrm{d}}$ Combined recreational and household activity as measured by the Cambridge index and shown as sex-specific categories of metabolic equivalents. ${ }^{\mathrm{e}}$ Diabetes based on self-reporting at baseline and ascertainment during follow-up.

\subsection{Dietary Intakes of AGEs and Mortality among CRC Patients}

The associations of CML, CEL, MG-H1, and the combined AGEs with CRC-specific and all-cause mortality are shown in Table 2. In our study population, the pre-diagnostic dietary intakes of AGEs were not statistically significantly associated with CRC-specific or all-cause mortality risk. For CRC-specific mortality, the fully adjusted HRs for the highest relative to the lowest quintile ( $\mathrm{HR}_{\mathrm{Q} 5}$ vs. $\left.\mathrm{Q} 1\right)$ of $\mathrm{CML}, \mathrm{CEL}, \mathrm{MG}-\mathrm{H} 1$, and the combined AGEs were $1.16\left(95 \%\right.$ CI: 0.98-1.36, $\left.P_{\text {trend }}=0.23\right), 1.11$ (95\% CI: $\left.0.94-1.31, P_{\text {trend }}=0.13\right)$, 1.10 (95\% CI: 0.94-1.28, $\left.P_{\text {trend }}=0.24\right)$, and 1.09 (95\% CI: 0.93-1.28, $\left.P_{\text {trend }}=0.29\right)$, respectively. For all-cause mortality, the fully adjusted $\mathrm{HR}_{\mathrm{Q} 5}$ vs. $\mathrm{Q} 1$ of CML, CEL, MG-H1, and the combined AGEs were 1.13 (95\% CI: 0.98-1.30, $\left.P_{\text {trend }}=0.36\right), 1.03$ (95\% CI: $0.89-1.19, P_{\text {trend }}=0.47$ ), 1.09 (95\% CI: 0.95-1.25, $\left.P_{\text {trend }}=0.23\right)$, and 1.08 (95\% CI: 0.94-1.24, $\left.P_{\text {trend }}=0.33\right)$, respectively. Although the suggestive positive associations for MG-H1 and combined AGEs were observed mostly among women, no statistically significant interactions by sex were identified (Supplemental Table S1). $p$-values for nonlinearity tests from the restricted cubic splines models were generally consistent with a linear response (Supplemental Figures S1 and S2), except for CML, which were consistent with a non-linear response.

Table 2. The associations of pre-diagnostic energy-adjusted dietary intakes of advanced glycation end products (AGEs) ${ }^{a}$ with all-cause and CRC-specific mortality among CRC patients in the EPIC study $(n=5801)$.

\begin{tabular}{|c|c|c|c|c|c|c|c|c|}
\hline \multirow{2}{*}{ AGEs ${ }^{a}$} & \multirow{2}{*}{ Cut-Offs } & \multirow{2}{*}{$N$} & \multicolumn{3}{|c|}{ All-Cause Mortality } & \multicolumn{3}{|c|}{ CRC-Specific Mortality } \\
\hline & & & Event & HR $(95 \% \mathrm{CI})^{\mathrm{b}, \mathrm{c}}$ & HR $(95 \% C I)^{b, d}$ & Event & HR $(95 \% C I){ }^{b, c}$ & HR $(95 \% C I)^{b, d}$ \\
\hline \multicolumn{9}{|l|}{ CML, mg/d } \\
\hline Quintile 1 & $<2.3$ & 1160 & 447 & 1.00 (ref) & 1.00 (ref) & 348 & 1.00 (ref) & 1.00 (ref) \\
\hline Quintile 2 & {$[2.3-2.7)$} & 1160 & 489 & $1.11(0.97-1.26)$ & $1.14(1.00-1.30)$ & 384 & $1.13(0.98-1.31)$ & $1.13(0.98-1.32)$ \\
\hline Quintile 3 & {$[2.7-3.1)$} & 1161 & 498 & $1.11(0.97-1.27)$ & $1.13(0.99-1.30)$ & 368 & $1.08(0.93-1.26)$ & $1.10(0.94-1.28)$ \\
\hline Quintile 4 & {$[3.1-3.7)$} & 1160 & 474 & $0.99(0.86-1.14)$ & $1.02(0.89-1.18)$ & 361 & $1.03(0.88-1.21)$ & $1.04(0.89-1.22)$ \\
\hline Quintile 5 & $\geq 3.7$ & 1160 & 513 & $1.08(0.93-1.25)$ & $1.13(0.98-1.30)$ & 380 & $1.14(0.97-1.34)$ & $1.16(0.98-1.36)$ \\
\hline$p_{\text {trend }} \mathrm{e}$ & & & & 0.72 & 0.36 & & 0.31 & 0.23 \\
\hline Per 1.0 & $g / d$ & & & $1.00(0.96,1.04)$ & $1.02(0.98-1.06)$ & & $1.03(0.98-1.08)$ & $1.03(0.98,1.08)$ \\
\hline \multicolumn{9}{|l|}{$\mathrm{CEL}, \mathrm{mg} / \mathrm{d}$} \\
\hline Quintile 1 & $<1.6$ & 1160 & 463 & 1.00 (ref) & 1.00 (ref) & 354 & 1.00 (ref) & 1.00 (ref) \\
\hline Quintile 2 & {$[1.6-1.9)$} & 1160 & 435 & $0.90(0.79-1.03)$ & $0.92(0.80-1.05)$ & 335 & $0.92(0.79-1.07)$ & $0.93(0.80-1.09)$ \\
\hline Quintile 3 & {$[1.9-2.2)$} & 1161 & 492 & $1.01(0.88-1.15)$ & $1.01(0.89-1.15)$ & 378 & $1.04(0.89-1.21)$ & $1.04(0.89-1.21)$ \\
\hline Quintile 4 & {$[2.2-2.6)$} & 1160 & 496 & $0.92(0.80-1.06)$ & $0.92(0.80-1.06)$ & 368 & $0.96(0.82-1.13)$ & $0.96(0.82-1.13)$ \\
\hline Quintile 5 & $\geq 2.6$ & 1160 & 535 & $1.03(0.89-1.18)$ & $1.03(0.89-1.19)$ & 406 & $1.10(0.93-1.29)$ & $1.11(0.94-1.31)$ \\
\hline$p_{\text {trend }} \mathrm{e}$ & & & & 0.45 & 0.47 & & 0.14 & 0.13 \\
\hline Per 0.7 & $\mathrm{~g} / \mathrm{d}$ & & & $1.01(0.97-1.06)$ & $1.01(0.97-1.06)$ & & $1.02(0.97-1.08)$ & $1.02(0.97-1.08)$ \\
\hline
\end{tabular}


Table 2. Cont.

\begin{tabular}{|c|c|c|c|c|c|c|c|c|}
\hline \multirow{2}{*}{ AGEs $^{a}$} & \multirow{2}{*}{ Cut-Offs } & \multirow{2}{*}{$N$} & \multicolumn{3}{|c|}{ All-Cause Mortality } & \multicolumn{3}{|c|}{ CRC-Specific Mortality } \\
\hline & & & Event & HR $(95 \% \mathrm{CI}){ }^{b, c}$ & HR $\left(95 \%\right.$ CI) ${ }^{b, d}$ & Event & HR $(95 \%$ CI) $b, c$ & HR $\left(95 \%\right.$ CI) ${ }^{b, d}$ \\
\hline \multicolumn{9}{|l|}{$\begin{array}{c}\text { MG-H1, } \\
\text { mg/d }\end{array}$} \\
\hline Quintile 1 & $<15.5$ & 1160 & 457 & 1.00 (ref) & 1.00 (ref) & 360 & 1.00 (ref) & 1.00 (ref) \\
\hline Quintile 2 & {$[15.5-18.4)$} & 1160 & 471 & $1.07(0.93-1.22)$ & $1.07(0.94-1.22)$ & 370 & $1.07(0.93-1.25)$ & $1.08(0.93-1.26)$ \\
\hline Quintile 3 & [18.4-21.4) & 1161 & 460 & $0.99(0.87-1.14)$ & $1.00(0.88-1.15)$ & 335 & $0.94(0.80-1.09)$ & $0.95(0.81-1.11)$ \\
\hline Quintile 4 & {$[21.4-26.1)$} & 1160 & 506 & $1.08(0.94-1.23)$ & $1.09(0.95-1.24)$ & 393 & $1.07(0.92-1.25)$ & $1.09(0.93-1.27)$ \\
\hline Quintile 5 & $\geq 26.1$ & 1160 & 527 & $1.07(0.93-1.22)$ & $1.09(0.95-1.25)$ & 383 & $1.08(0.92-1.26)$ & $1.10(0.94-1.28)$ \\
\hline$p_{\text {trend }} \mathrm{e}$ & & & & 0.37 & 0.23 & & 0.35 & 0.24 \\
\hline \multicolumn{2}{|c|}{ Per $8.47 \mathrm{mg} / \mathrm{d}$} & & & $1.01(0.97-1.05)$ & $1.02(0.98-1.05)$ & & $1.02(0.98-1.07)$ & $1.03(0.98-1.08)$ \\
\hline \multicolumn{9}{|c|}{ Combined AGEs ${ }^{\mathrm{f}}, \mathrm{mg} / \mathrm{d}$} \\
\hline Quintile 1 & $<19.8$ & 1160 & 453 & 1.00 (ref) & 1.00 (ref) & 356 & 1.00 (ref) & 1.00 (ref) \\
\hline Quintile 2 & {$[19.8-23.2)$} & 1160 & 467 & $1.08(0.94-1.23)$ & $1.09(0.95-1.24)$ & 367 & $1.08(0.93-1.26)$ & $1.10(0.95-1.28)$ \\
\hline Quintile 3 & {$[23.2-26.8)$} & 1161 & 463 & $1.01(0.88-1.15)$ & $1.02(0.89-1.17)$ & 336 & $0.96(0.82-1.12)$ & $0.97(0.83-1.13)$ \\
\hline Quintile 4 & {$[26.8-32.3)$} & 1160 & 520 & $1.09(0.95-1.24)$ & $1.10(0.96-1.26)$ & 405 & $1.10(0.95-1.28)$ & $1.11(0.96-1.30)$ \\
\hline Quintile 5 & $\geq 32.3$ & 1160 & 518 & $1.06(0.92-1.21)$ & $1.08(0.94-1.24)$ & 377 & $1.07(0.91-1.25)$ & $1.09(0.93-1.28)$ \\
\hline$p_{\text {trend }} \mathrm{e}$ & & & & 0.50 & 0.33 & & 0.42 & 0.29 \\
\hline Per 9. & $\mathrm{~g} / \mathrm{d}$ & & & $1.01(0.97-1.05)$ & $1.02(0.98-1.05)$ & & $1.02(0.98-1.08)$ & $1.03(0.98-1.08)$ \\
\hline
\end{tabular}

Abbreviations: AGE, advanced glycation end products; CRC, colorectal cancer; CML, Ne-(caroxymethyl)lysine; CEL, Ne-(1caroxyethyl)lysine; d, day; MGH1, Ne-(5-hydro-5-methyl-4-imidazolon-2-yl)-ornithine; mg, milligram; HR, hazard ratio; ref, reference category. ${ }^{a}$ Residuals were computed by a linear regression of the log-transformed intake of AGEs on total energy intake, sex and center. ${ }^{b}$ Quintile 1 was a reference category in each model. ${ }^{c}$ Multivariable cox proportional hazard model, stratified by center, and adjusted for sex, age at diagnosis (yrs; continuous) and stage (categorical) and total energy intake (kcal/d; continuous). ${ }^{\mathrm{d}}$ Multivariable cox proportional hazard model, stratified by center, and adjusted for sex, age at diagnosis (y; continuous), stage (categorical), total energy intake (kcal/d; continuous), year of diagnosis (continuous), location of tumor(categorical), BMI (continuous), smoking status(categorical) and prevalent/incident diabetes (categorical). ${ }^{e} P_{\text {trend }}$ was calculated with the median value of each quintile of AGE as a continuous variable, adjusted for covariates in the corresponding model. ${ }^{\mathrm{f}}$ Combined AGEs: CML+CEL+MGH1.

\subsection{Sensitivity Analyses}

Excluding participants with missing stage information (around 26\%) or using imputed missing tumor stage data had no effect on the results (Supplemental Table S2) We also assessed whether the association between dietary AGEs and risk of mortality differed for long-term survivors. After the exclusion of cases that occurred during the first 5 years of follow-up, a positive association of pre-diagnostic dietary AGEs with CRC-specific mortality was found for CML (HR $=1.19,95 \% \mathrm{CI}$ : 1.04-1.36; $\left.P_{\text {trend }}=0.01\right)$ and CEL $(\mathrm{HR}=1.15$, 95\% CI: 1.02-1.30; $\left.P_{\text {trend }}=0.02\right)$ (Supplemental Table S2). Similar results were also observed for all-cause mortality (Supplemental Table S3).

\subsection{Stratified Analyses}

Stratified analyses suggested differences in the associations between dietary AGEs and CRCspecific (Figure 1 and Supplemental Table S2) and all-cause mortality (Supplemental Table S3) across select subcategories of potential a priori defined biologically plausible effect modifiers. Statistically significant positive associations between AGEs and mortality risk were observed among participants who were diagnosed with CRC more than 11 years after recruitment (CRC-specific mortality, per one SD change in AGE; $\mathrm{HR} \mathrm{CML}_{\mathrm{C}}=1.09,95 \% \mathrm{CI}$ : 1.00-1.20; $\mathrm{HR}_{\mathrm{CEL}}=1.11,95 \% \mathrm{CI}: 1.02-1.20, \mathrm{HR}_{\mathrm{MG}-\mathrm{H} 1}=1.11,95 \% \mathrm{CI}: 1.02-1.21$, and HR AGEs $=1.11,95 \%$ CI: $1.03-1.21$; all $P_{\text {interaction }}<0.01$ ). There were also some indications that the associations of CML, CEL, MG-H1, and the combined AGEs were slightly stronger among participants without type II diabetes, with HRs of 1.07 (95\% CI: 1.01-1.13), 1.05 (95\% CI: $1.00-1.12), 1.06$ (95\% CI: 1.00-1.12), and 1.06 (95\% CI: 1.00-1.12) for CRC-specific mortality, and 1.05 (95\% CI: 1.00-1.11), 1.04 (95\% CI: 1.00-1.09), 1.05 (95\% CI: 1.00-1.10), and 1.05 (95\% CI: 1.00-1.11) for all-cause mortality, respectively. 


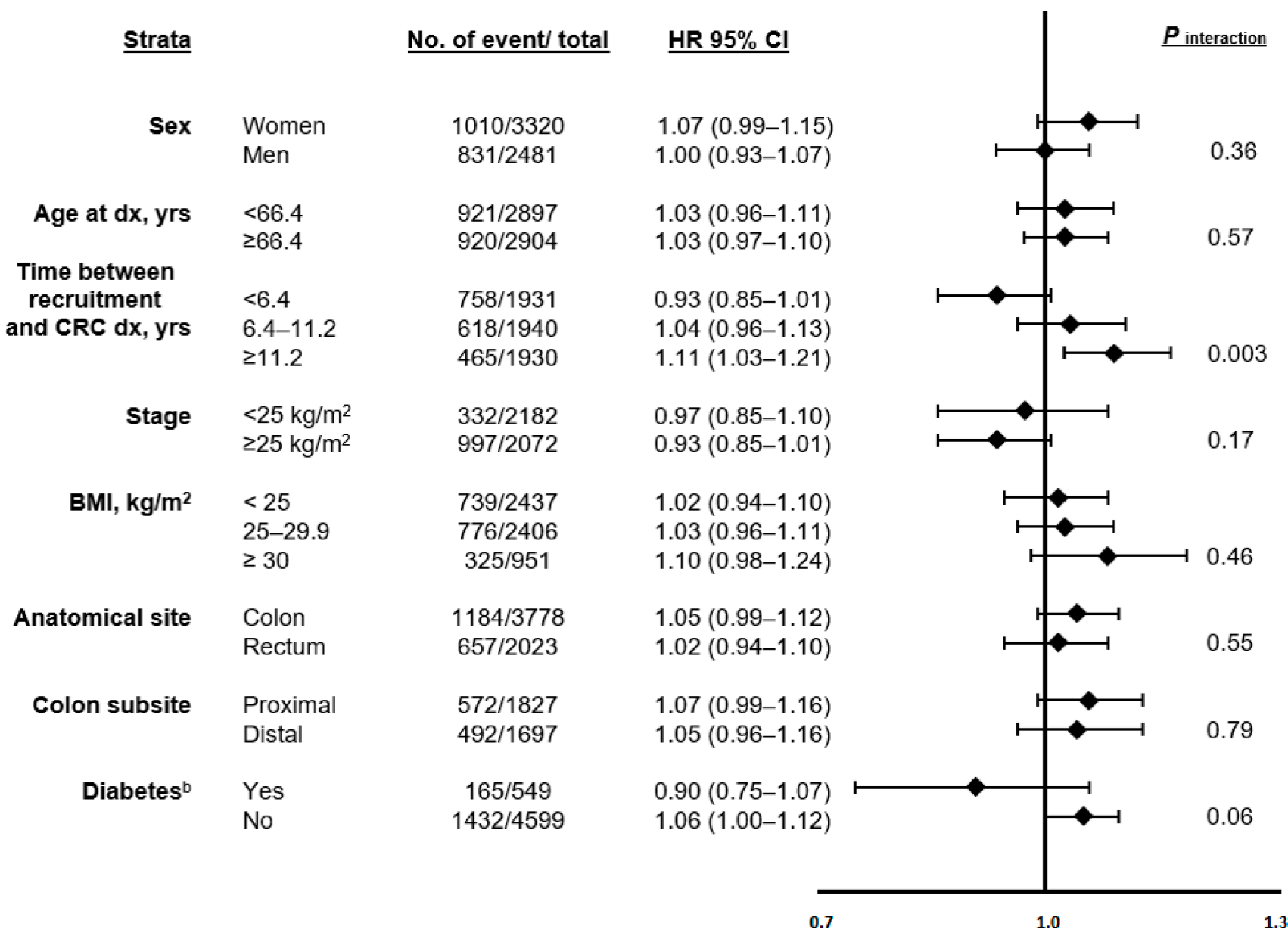

Figure 1. The associations of pre-diagnostic combined dietary intake of AGEs a with CRC-specific mortality across strata of selected patient characteristics among individuals with CRC in the EPIC study $(n=5801)$. Abbreviations: AGE, advanced glycation end products; CRC, colorectal cancer. ${ }^{a}$ Combined AGEs: CML+CEL+MGH1. ${ }^{\mathrm{b}}$ Diabetes based on self-reporting at baseline and ascertainment during follow-up.

\section{Discussion}

Our findings suggest that pre-diagnostic dietary intakes of AGEs were not associated with CRC-specific and all-cause mortality among CRC patients in this large prospective study. Stratified analyses suggested potential interactions by time between recruitment and CRC diagnosis and that the association might be limited to individuals without type II diabetes and with more than 5 years of follow-up after cancer diagnosis.

AGEs are associated with oxidative stress and inflammation [5,9], which can be involved in the initiation and progression of multiple chronic diseases, including cancers [11-13]. The accumulation of AGEs can activate intracellular signals via binding to RAGEs, which in turn can promote inflammation and tissue injury sustained by a RAGE-dependent expression of proinflammatory mediators, such as circulating monocyte chemotactic protein 1 (MCP-1) and vascular cell adhesion molecule 1 (VCAM-1) [5] Exposure to AGEs can also lead to increased levels of reactive oxygen species, which are associated with an increase in oxidative stress. Both inflammation and oxidative stress are associated with cellular and DNA damage, which could lead to carcinogenic mutation and subsequent initiation, development, and progression of CRC [42-44]. In addition, AGEs might crosslink with the proteins, leading to functional alterations of the vasculature and angiogenesis [17,18], which might contribute to carcinogenesis [45]. AGEs could also be associated with adverse outcomes, including recurrence and death, among CRC survivors. Evidence from previous clinical trials has linked an AGE-restricted diet to a decrease in 
plasma AGEs and to markers of oxidative stress and inflammation $[13,15,46]$, which might support the idea that dietary AGEs contribute to the pool of AGEs in the human body and could lead to CRC development and progression.

Only two previous epidemiologic studies investigated the association of dietary AGE intakes with all-cause or cause-specific mortality risk and were limited to only dietary CML $[25,31]$. A prospective study among postmenopausal women diagnosed with invasive breast cancer $(n=2073)$ in the Women's Health Initiative (WHI) study reported a statistically significant positive association between higher post-diagnosis dietary intake of CML and an increased risk of all-cause (HR $=1.51,95 \%$ CI: 1.17-1.94), CVD (HR $=1.86,95 \%$ CI: 1.19-2.91), and breast cancer ( $\mathrm{HR}=2.14,95 \%$ CI: 1.19-3.84) mortality [25]. However, the investigators measured CML only, and the assessment of dietary CML was based on food composition as determined via an enzyme-linked immunosorbent assay (ELISA) method, which is different from our quantitative instrumental mass-spectrometry method for assessing dietary AGEs. The other study in a Japanese prospective cohort (men $=13,335$ and women $=15,724$ ) suggested that dietary CML intake estimated using a reference liquid chromatography tandem mass-spectrometry-based data set was not statistically significantly associated with higher mortality in healthy adults [31]. However, among men, higher CML intake was associated with lower risk for all-cause mortality ( $\mathrm{HR}=0.89$, 95\% CI: 0.79-1.00; $P_{\text {trend }}=0.05$ ). Considering differences in timing of exposure (after cancer diagnosis in the WHI study), AGE assessment methods (ELISA), and study populations (breast cancer survivors and healthy adults), our results among patients with CRC may not be directly comparable with previous findings. However, somewhat consistent with previous findings, our study suggested that the positive AGE-mortality association might be stronger among women. A prospective cohort study from Finland of non-diabetic men $(n=535)$ and women $(n=606)$ also reported a stronger positive association between AGEmodified bovine serum albumin (BSA), measured via ELISA, and all-cause mortality in women but not men [30]. The reason behind this difference is not clear, but it is possible that the biological differences between men and women in metabolism and immune response could account for both our and previous findings. Earlier evidence suggested that AGE accumulation is associated with positive expression of estrogen receptor alpha among breast cancer patients and supported a potential mechanistic link between AGEs and estrogen signaling $[47,48]$. This potential mechanism might contribute to the stronger AGEmortality associations in women with CRC, although we did not observe any differences in women by menopausal status.

In our sensitivity analyses, after restricting participants to those who were diagnosed with CRC after 11 years of recruitment, positive associations of dietary AGEs with increased mortality risk were observed. These results are consistent with our original hypotheses. A possibility for this could be that AGEs might influence molecular features and specific molecular pathways of colon cancer during its development and progression [49], so that earlier AGE exposures may be more important than later ones in relation to mortality risk. We also found a suggestive positive association between intakes of CML and CEL and high mortality risk with more than two years of follow-up after cancer diagnosis, which suggests that the exposure might be more relevant for long-term outcomes. We also found a positive association between intake of AGEs and high mortality risk in participants without type II diabetes, which could in part be due to higher formation of endogenous AGEs relative to dietary intake of AGEs among individuals with type II diabetes [9].

The major strengths of this study include the prospective study design, large sample size, comprehensive collection and assessment of multiple potential confounding/effect modifying factors, and multiple sensitivity analyses. In addition, our study used a state-ofthe-art quantitative instrumental mass-spectrometry-based method to assess three different types of AGEs in foods [35], and we followed the recent "quality control" recommendations for studies on AGEs, i.e., the study of several specific AGEs and the use of a validated food composition database to estimate individual dietary AGE exposures [50]. 
Our study also has several limitations. First, the dietary data were measured at baseline before cancer diagnosis, and some participants might have modified their diet after CRC diagnosis. However, earlier (not later) exposure to AGEs could be more important in shaping the microenvironment for tumor development and determining its aggressiveness. Second, we cannot exclude a potential misclassification in estimating dietary AGEs, which is also influenced by personal cooking preferences. However, it was reported previously that higher intakes of CEL, CML, and MG-H1 were positively associated with weight gain and obesity after, on average, 5 years of follow-up in the EPIC study, which indicates the validity of our dietary AGE assessment [36]. Third, our study population was limited to Western European CRC patients, which restricts the generalizability of the study results. Fourth, CRC cases might have changed their diet after cancer diagnosis. However, the prediagnostic diet could be more indicative of exposures that promoted tumor development and progression and influenced the tumor molecular profile and aggressiveness. Fifth, we did not have information on CRC treatment. Generally, during the follow-up period, we would not expect CRC treatment to differ by country within the countries and centers participating in this study or by year of diagnosis or tumor stage. Therefore, our analyses were conducted stratified by country of CRC diagnosis and adjusted for tumor stage and year of diagnosis. Finally, to estimate the effect of missing CRC stage data we used several approaches, all of which demonstrated the robustness of effect estimates against uncertainties in CRC stage classification.

\section{Conclusions}

In conclusion, our findings suggest that pre-diagnostic dietary intakes of AGEs might not be associated with CRC-specific or all-cause mortality among individuals diagnosed with CRC. Further studies are necessary to investigate these associations in different populations and examine whether these associations are stronger among women and long-term survivors and differ by diabetes status and timing of exposure to AGEs.

Supplementary Materials: The following are available online at https://www.mdpi.com/article/ $10.3390 /$ nu13124435/s1, Table S1: The associations of pre-diagnostic dietary energy-adjusted intakes of advanced glycation end products (AGEs) with all-cause and CRC mortality by sex and among men and women with CRC combined for sex-specific quintiles and per one SD change, the EPIC study $(n=5801)$, Table S2: Adjusted HRs and 95\% CIs for an increment of per one SD change of dietary advanced glycation end products (AGEs) and CRC-specific mortality across strata of potential effect modifiers among CRC patients in the EPIC study $(n=5801)$, Table S3: Adjusted HRs and $95 \%$ CIs for an increment of per one SD change of dietary advanced glycation end products (AGEs) and all-cause mortality across strata of potential effect modifiers among CRC patients in the EPIC study $(n=5801)$, Figure S1: Non-parametrical restricted cubic splines of AGEs with CRC-specific mortality among CRC patients in the EPIC study, Figure S2: Non-parametrical restricted cubic splines of AGEs with all-cause mortality among CRC patients in the EPIC study.

Author Contributions: Conceptualization, V.F. and M.J.; formal analysis, Z.M. and Z.Z.; methodology, M.J. and H.F.; data curation, M.J. and E.K.A.; investigation, all co-authors; resources, all co-authors; writing — original draft preparation, Z.M. and V.F.; writing—review and editing, all co-authors; supervision, V.F.; project administration, V.F. and M.J.; funding, M.J. All co-authors reviewed and approved the manuscript and commented on the analysis and interpretation of the findings. All authors have read and agreed to the published version of the manuscript.

Funding: Grant support: Funding [WCRF 2015/1391, PI: M. Jenab] was obtained from Wereld Kanker Onderzoek Fonds (WKOF), as part of the World Cancer Research Fund International grant program. The coordination of EPIC is financially supported by International Agency for Research on Cancer (IARC) and also by the Department of Epidemiology and Biostatistics, School of Public Health, Imperial College London, which has additional infrastructure support provided by the NIHR Imperial Biomedical Research Centre (BRC). The national cohorts are supported by: Danish Cancer Society (Denmark); Ligue Contre le Cancer, Institut Gustave Roussy, Mutuelle Générale de l'Education Nationale, Institut National de la Santé et de la Recherche Médicale (INSERM) (France); German Cancer Aid, German Cancer Research Center (DKFZ), German Institute of Human Nutrition Potsdam- 
Rehbruecke (DIfE), Federal Ministry of Education and Research (BMBF) (Germany); Associazione Italiana per la Ricerca sul Cancro-AIRC-Italy, Compagnia di SanPaolo and National Research Council (Italy); Dutch Ministry of Public Health, Welfare and Sports (VWS), Netherlands Cancer Registry (NKR), LK Research Funds, Dutch Prevention Funds, Dutch ZON (Zorg Onderzoek Nederland), World Cancer Research Fund (WCRF), Statistics Netherlands (The Netherlands); Health Research Fund (FIS) - Instituto de Salud Carlos III (ISCIII), Regional Governments of Andalucía, Asturias, Basque Country, Murcia and Navarra, the Catalan Institute of Oncology-ICO (Spain); Swedish Cancer Society, Swedish Research Council and County Councils of Skåne and Västerbotten (Sweden); Cancer Research UK (14136 to EPIC-Norfolk; C8221/ A29017 to EPIC-Oxford), Medical Research Council (1000143 to EPIC-Norfolk; MR/M012190/1 to EPIC-Oxford) (United Kingdom). The EPICNorfolk study (DOI 10.22025/2019.10.105.00004) received funding from the Medical Research Council (MR/N003284/1 and MC-UU_12015/1) and Cancer Research UK (C864/A14136). V. Fedirko is supported by the Cancer Prevention and Research Institute of Texas (CPRIT) Rising Stars Award (Grant ID RR200056). The funders had no role in study design, data collection and analysis, decision to publish, or preparation of the manuscript.

Institutional Review Board Statement: The study was conducted according to the guidelines of the Declaration of Helsinki and approved by the Ethical Committee of the International Agency for Research on Cancer (IARC) and local ethics committees pertaining to EPIC Centers. The EPIC cohort was successfully reevaluated by the International Agency for Research on Cancer Ethics Committee in 2017. The current study received further approval by the IARC Ethics Committee (IEC Project No. 18-10).

Informed Consent Statement: Written consent was obtained from all subjects involved in the study.

Data Availability Statement: Data supporting reported results are available from the corresponding author upon request.

Acknowledgments: We are grateful to all the participants who were part of the project and to the many members of the study teams at the University of Cambridge and the EPIC center in Florence who enabled this research.

Conflicts of Interest: The authors declare no conflict of interest.

Disclaimer: Where authors are identified as personnel of the International Agency for Research on Cancer/World Health Organization, the authors alone are responsible for the views expressed in this article and they do not necessarily represent the decisions, policy, or views of the International Agency for Research on Cancer/World Health Organization. The funding sources had no influence on the design of the study; the collection, analysis, and interpretation of data; the writing of the report; or the decision to submit the paper for publication.

\section{References}

1. Cancer Today. Available online: https://gco.iarc.fr/today/home (accessed on 23 September 2021).

2. Potter, J.D.; Slattery, M.L.; Bostick, R.M.; Gapstur, S.M. Colon Cancer: A Review of the Epidemiology. Epidemiol. Rev. 1993, 15, 499-545. [CrossRef]

3. Siegel, R.L.; Miller, K.D.; Jemal, A. Cancer statistics, 2020. CA Cancer J. Clin. 2020, 70, 7-30. [CrossRef]

4. Clinton, S.K.; Giovannucci, E.L.; Hursting, S.D. The World Cancer Research Fund/American Institute for Cancer Research Third Expert Report on Diet, Nutrition, Physical Activity, and Cancer: Impact and Future Directions. J. Nutr. 2020, 150, 663-671. [CrossRef]

5. Uribarri, J.; Del Castillo, M.D.; De La Maza, M.P.; Filip, R.; Gugliucci, A.; Luevano-Contreras, C.; Macías-Cervantes, M.H.; Bastos, D.H.M.; Medrano, A.; Menini, T.; et al. Dietary Advanced Glycation End Products and Their Role in Health and Disease. Adv. Nutr. 2015, 6, 461-473. [CrossRef] [PubMed]

6. Ahmed, N. Advanced glycation endproducts-Role in pathology of diabetic complications. Diabetes Res. Clin. Pract. 2005, 67, 3-21. [CrossRef] [PubMed]

7. O'Brien, J.; Morrissey, P.A.; Ames, J.M. Nutritional and toxicological aspects of the Maillard browning reaction in foods. Crit. Rev. Food Sci. Nutr. 1989, 28, 211-248. [CrossRef] [PubMed]

8. Cerami, C.; Founds, H.; Nicholl, I.; Mitsuhashi, T.; Giordano, D.; Vanpatten, S.; Lee, A.; Al-Abed, Y.; Vlassara, H.; Bucala, R.; et al. Tobacco smoke is a source of toxic reactive glycation products. Proc. Natl. Acad. Sci. USA 1997, 94, 13915-13920. [CrossRef]

9. Poulsen, M.W.; Hedegaard, R.V.; Andersen, J.M.; de Courten, B.; Bügel, S.; Nielsen, J.; Skibsted, L.H.; Dragsted, L.O. Advanced glycation endproducts in food and their effects on health. Food Chem. Toxicol. 2013, 60, 10-37. [CrossRef] [PubMed] 
10. Scheijen, J.L.; Clevers, E.; Engelen, L.; Dagnelie, P.C.; Brouns, F.; Stehouwer, C.D.; Schalkwijk, C.G. Analysis of advanced glycation endproducts in selected food items by ultra-performance liquid chromatography tandem mass spectrometry: Presentation of a dietary AGE database. Food Chem. 2016, 190, 1145-1150. [CrossRef]

11. Cai, W.; Gao, Q.-D.; Zhu, L.; Peppa, M.; He, C.; Vlassara, H. Oxidative Stress-Inducing Carbonyl Compounds From Common Foods: Novel Mediators of Cellular Dysfunction. Mol. Med. 2002, 8, 337-346. [CrossRef]

12. Vlassara, H.; Cai, W.; Tripp, E.; Pyzik, R.; Yee, K.; Goldberg, L.; Tansman, L.; Chen, X.; Mani, V.; Fayad, Z.A.; et al. Oral AGE restriction ameliorates insulin resistance in obese individuals with the metabolic syndrome: A randomised controlled trial. Diabetologia 2016, 59, 2181-2192. [CrossRef] [PubMed]

13. Vlassara, H.; Cai, W.; Crandall, J.; Goldberg, T.; Oberstein, R.; Dardaine, V.; Peppa, M.; Rayfield, E.J. Nonlinear partial differential equations and applications: Inflammatory mediators are induced by dietary glycotoxins, a major risk factor for diabetic angiopathy. Proc. Natl. Acad. Sci. USA 2002, 99, 15596-15601. [CrossRef] [PubMed]

14. Takeuchi, M. Serum Levels of Toxic AGEs (TAGE) May Be a Promising Novel Biomarker for the Onset/Progression of LifestyleRelated Diseases. Diagnostics 2016, 6, 23. [CrossRef] [PubMed]

15. Vlassara, H.; Cai, W.; Goodman, S.; Pyzik, R.; Yong, A.; Chen, X.; Zhu, L.; Neade, T.; Beeri, M.; Silverman, J.M.; et al. Protection against Loss of Innate Defenses in Adulthood by Low Advanced Glycation End Products (AGE) Intake: Role of the Antiinflammatory AGE Receptor-1. J. Clin. Endocrinol. Metab. 2009, 94, 4483-4491. [CrossRef]

16. Uribarri, J.; Cai, W.; Peppa, M.; Goodman, S.; Ferrucci, L.; Striker, G.; Vlassara, H. Circulating Glycotoxins and Dietary Advanced Glycation Endproducts: Two Links to Inflammatory Response, Oxidative Stress, and Aging. J. Gerontol. Ser. A Boil. Sci. Med. Sci. 2007, 62, 427-433. [CrossRef]

17. Greenwald, S. Ageing of the conduit arteries. J. Pathol. 2007, 211, 157-172. [CrossRef] [PubMed]

18. Singh, R.; Barden, A.; Mori, T.; Beilin, L. Advanced glycation end-products: A review. Diabetologia 2001, 44, 129-146. [CrossRef] [PubMed]

19. Arsov, S.; Graaff, R.; Van Oeveren, W.; Stegmayr, B.; Sikole, A.; Rakhorst, G.; Smit, A.J. Advanced glycation end-products and skin autofluorescence in end-stage renal disease: A review. Clin. Chem. Lab. Med. 2014, 52, 11-20. [CrossRef]

20. Hartog, J.W.L.; Voors, A.A.; Bakker, S.J.L.; Smit, A.J.; Van Veldhuisen, D.J. Advanced glycation end-products (AGEs) and heart failure: Pathophysiology and clinical implications. Eur. J. Heart Fail. 2007, 9, 1146-1155. [CrossRef]

21. Kizer, J.R.; Benkeser, D.; Arnold, A.M.; Ix, J.H.; Mukamal, K.J.; Djousse, L.; Tracy, R.P.; Siscovick, D.S.; Psaty, B.M.; Zieman, S.J. Advanced glycation/glycoxidation endproduct carboxymethyl-lysine and incidence of coronary heart disease and stroke in older adults. Atherosclerosis 2014, 235, 116-121. [CrossRef] [PubMed]

22. Di Pino, A.; Currenti, W.; Urbano, F.; Scicali, R.; Piro, S.; Purrello, F.; Rabuazzo, A.M. High intake of dietary advanced glycation end-products is associated with increased arterial stiffness and inflammation in subjects with type 2 diabetes. Nutr. Metab. Cardiovasc. Dis. 2017, 27, 978-984. [CrossRef] [PubMed]

23. Nowotny, K.; Schröter, D.; Schreiner, M.; Grune, T. Dietary advanced glycation end products and their relevance for human health. Ageing Res. Rev. 2018, 47, 55-66. [CrossRef] [PubMed]

24. Mayén, A.; Aglago, E.K.; Knaze, V.; Cordova, R.; Schalkwijk, C.G.; Wagner, K.; Aleksandrova, K.; Fedirko, V.; Keski-Rahkonen, P.; Leitzmann, M.F.; et al. Dietary intake of advanced glycation endproducts and risk of hepatobiliary cancers: A multinational cohort study. Int. J. Cancer 2021, 149, 854-864. [CrossRef] [PubMed]

25. Peterson, L.L.; Omofuma, O.; Turner, D.P.; Merchant, A.; Zhang, J.; Neuhouser, M.L.; Snetselaar, L.; Caan, B.J.; Shadyab, A.H.; Saquib, N.; et al. Dietary advanced glycation end products (AGEs) and breast cancer mortality in the women's health initiative (WHI). J. Clin. Oncol. 2020, 38, 1570-1570. [CrossRef]

26. Ebert, H.; Lacruz, M.E.; Kluttig, A.; Simm, A.; Greiser, K.H.; Tiller, D.; Kartschmit, N.; Mikolajczyk, R. Association between advanced glycation end products, their soluble receptor, and mortality in the general population: Results from the CARLA study. Exp. Gerontol. 2019, 131, 110815. [CrossRef] [PubMed]

27. Semba, R.D.; Ferrucci, L.; Sun, K.; Beck, J.; Dalal, M.; Varadhan, R.; Walston, J.; Guralnik, J.M.; Fried, L.P. Advanced glycation end products and their circulating receptors predict cardiovascular disease mortality in older community-dwelling women. Aging Clin. Exp. Res. 2009, 21, 182-190. [CrossRef]

28. Roberts, M.A.; Thomas, M.C.; Fernando, D.; Macmillan, N.; Power, D.A.; Ierino, F.L. Low molecular weight advanced glycation end products predict mortality in asymptomatic patients receiving chronic haemodialysis. Nephrol. Dial. Transplant. 2006, 21, 1611-1617. [CrossRef] [PubMed]

29. Nin, J.W.; Jorsal, A.; Ferreira, I.; Schalkwijk, C.G.; Prins, M.H.; Parving, H.-H.; Tarnow, L.; Rossing, P.; Stehouwer, C.D. Higher Plasma Levels of Advanced Glycation End Products Are Associated With Incident Cardiovascular Disease and All-Cause Mortality in Type 1 Diabetes: A 12-year follow-up study. Diabetes Care 2011, 34, 442-447. [CrossRef]

30. Kilhovd, B.K.; Juutilainen, A.; Lehto, S.; Ronnemaa, T.; Torjesen, P.A.; Birkeland, K.I.; Berg, T.J.; Hanssen, K.F.; Laakso, M. High Serum Levels of Advanced Glycation End Products Predict Increased Coronary Heart Disease Mortality in Nondiabetic Women but not in Nondiabetic Men. Arter. Thromb. Vasc. Biol. 2005, 25, 815-820. [CrossRef]

31. Nagata, C.; Wada, K.; Yamakawa, M.; Nakashima, Y.; Koda, S.; Uji, T.; Oba, S. Dietary Intake of Ne-carboxymethyl-lysine, a Major Advanced Glycation End Product, is Not Associated with Increased Risk of Mortality in Japanese Adults in the Takayama Study. J. Nutr. 2020, 150, 2799-2805. [CrossRef] 
32. Riboli, E.; Hunt, K.J.; Slimani, N.; Ferraria, P.; Norata, T.; Fahey, M.; Charrondierea, U.R.; Hemona, B.; Casagrandea, C.; Vignata, J.; et al. European Prospective Investigation into Cancer and Nutrition (EPIC): Study populations and data collection. Public Health Nutr. 2002, 5, 1113-1124. [CrossRef] [PubMed]

33. Bingham, S.; Riboli, E. Diet and cancer - the European Prospective Investigation into Cancer and Nutrition. Nat. Rev. Cancer 2004, 4, 206-215. [CrossRef] [PubMed]

34. Slimani, N.; Deharveng, G.; Unwin, I.; Southgate, D.A.T.; Vignat, J.; Skeie, G.; Salvini, S.; Parpinel, M.; Møller, A.; Ireland, J.; et al. The EPIC nutrient database project (ENDB): A first attempt to standardize nutrient databases across the 10 European countries participating in the EPIC study. Eur. J. Clin. Nutr. 2007, 61, 1037-1056. [CrossRef] [PubMed]

35. Aglago, E.K.; Mayén, A.-L.; Knaze, V.; Freisling, H.; Fedirko, V.; Hughes, D.J.; Jiao, L.; Eriksen, A.K.; Tjønneland, A.; BoutronRuault, M.-C.; et al. Dietary Advanced Glycation End-Products and Colorectal Cancer Risk in the European Prospective Investigation into Cancer and Nutrition (EPIC) Study. Nutrients 2021, 13, 3132. [CrossRef]

36. Cordova, R.; Knaze, V.; Viallon, V.; Rust, P.; Schalkwijk, C.G.; Weiderpass, E.; Wagner, K.-H.; Mayen-Chacon, A.-L.; Aglago, E.K.; Dahm, C.; et al. Dietary intake of advanced glycation end products (AGEs) and changes in body weight in European adults. Eur. J. Nutr. 2019, 59, 2893-2904. [CrossRef] [PubMed]

37. Willett, W.C.; Howe, G.R.; Kushi, L.H. Adjustment for total energy intake in epidemiologic studies. Am. J. Clin. Nutr. 1997, 65, 1220S-1228S. [CrossRef] [PubMed]

38. Durrleman, S.; Simon, R. Flexible regression models with cubic splines. Stat. Med. 1989, 8, 551-561. [CrossRef]

39. Govindarajulu, U.S.; Spiegelman, D.; Thurston, S.W.; Ganguli, B.; Eisen, E.A. Comparing smoothing techniques in Cox models for exposure-response relationships. Stat. Med. 2007, 26, 3735-3752. [CrossRef] [PubMed]

40. Li, R.; Hertzmark, E.; Louie, M.; Chen, L.; Spiegelman, D. The SAS LGTPHCURV9 Macro; Channing Laboratory: Boston, MA, USA, 2011.

41. Zhang, S.; Li, F.; Younes, M.; Liu, H.; Chen, C.; Yao, Q. Reduced Selenium-Binding Protein 1 in Breast Cancer Correlates with Poor Survival and Resistance to the Anti-Proliferative Effects of Selenium. PLoS ONE 2013, 8, e63702. [CrossRef]

42. Reuter, S.; Gupta, S.C.; Chaturvedi, M.M.; Aggarwal, B.B. Oxidative stress, inflammation, and cancer: How are they linked? Free. Radic. Biol. Med. 2010, 49, 1603-1616. [CrossRef] [PubMed]

43. Stone, W.L.; Krishnan, K.; E Campbell, S.; E Palau, V. The role of antioxidants and pro-oxidants in colon cancer. World J. Gastrointest. Oncol. 2014, 6, 55-66. [CrossRef] [PubMed]

44. Chen, J.; Pitmon, E.; Wang, K. Microbiome, inflammation and colorectal cancer. Semin. Immunol. 2017, 32, 43-53. [CrossRef]

45. Levental, K.; Yu, H.; Kass, L.; Lakins, J.N.; Egeblad, M.; Erler, J.; Fong, S.F.; Csiszar, K.; Giaccia, A.; Weninger, W.; et al. Matrix Crosslinking Forces Tumor Progression by Enhancing Integrin Signaling. Cell 2009, 139, 891-906. [CrossRef]

46. Uribarri, J.; Peppa, M.; Cai, W.; Goldberg, T.; Lu, M.; He, C.; Vlassara, H. Restriction of Dietary Glycotoxins Reduces Excessive Advanced Glycation End Products in Renal Failure Patients. J. Am. Soc. Nephrol. 2003, 14, 728-731. [CrossRef] [PubMed]

47. Walter, K.; Ford, M.E.; Gregoski, M.J.; Kramer, R.M.; Knight, K.D.; Spruill, L.; Nogueira, L.M.; Krisanits, B.A.; Phan, V.; La Rue, A.C.; et al. Advanced glycation end products are elevated in estrogen receptor-positive breast cancer patients, alter response to therapy, and can be targeted by lifestyle intervention. Breast Cancer Res. Treat. 2018, 173, 559-571. [CrossRef]

48. Nass, N.; Ignatov, A.; Andreas, L.; Weißenborn, C.; Kalinski, T.; Sel, S. Accumulation of the advanced glycation end product carboxymethyl lysine in breast cancer is positively associated with estrogen receptor expression and unfavorable prognosis in estrogen receptor-negative cases. Histochem. Cell Biol. 2016, 147, 625-634. [CrossRef] [PubMed]

49. Lin, J.-A.; Wu, C.; Lu, C.; Hsia, S.; Yen, G. Glycative stress from advanced glycation end products (AGEs) and dicarbonyls: An emerging biological factor in cancer onset and progression. Mol. Nutr. Food Res. 2016, 60, 1850-1864. [CrossRef]

50. Hellwig, M.; Humpf, H.-U.; Hengstler, J.G.; Mally, A.; Vieths, S.; Henle, T. Quality Criteria for Studies on Dietary Glycation Compounds and Human Health. J. Agric. Food Chem. 2019, 67, 11307-11311. [CrossRef] [PubMed] 